\title{
REPORTS OF MEETINGS
}

\author{
1990 IEEE Ultrasonics Symposium
}

Sessions A, F, K, Q, V, and AA dealt with topics in ultrasonic dosimetry, tissue characterization and blood flow measurements. In the only oral session devoted to a discussion of ultrasonic dosimetry in this meeting, Mark Schaffer of Sonic Technologies proposed two alternatives to take into consideration of non-linear propagation effects that are not considered by the present FDA approach for estimating in situ intensity, N. Smith of University of Illinois described an automated system to assess bioeffects of ultrasound on rat fetus, Sidney Leeman(King's College, UK) presented results using a large aperture PVDF transducer to account for the difference in intensities measured by radiation pressure and calorimetry, and Kai Thomenious of Interspec, Inc gave an invited talk on thermal dosimetry models for diagnostic ultrasound in which the rationale for establishing a thermal index, various bio-heat transfer models and computer simulated results were presented. The results showed that the temperature rise in the tissues is critically dependent upon the curvature of the transducer and that thermal properties of the transducer needs also to be considered.

In the two tissue characterization sessions, M. Insana of University of Kansas, E. Verdonk of Washington University reported recent findings on the effects of anisotropic nature of kidney and myocardium on backscatter and sound velocity. Backscatter is in general maximal when ultrasonic beam is perpendicular to the tissue structure and minimal when parallel. The Washington University group however showed the behavior of ultrasound velocity is just the opposite, maximal when beam is parallel to the myocardial fibers and minimal when perpendicular. G. Brandenburg(Osteo Technology) and H. Asai of Tohoku University, Japan described clinical results using a dedicated instrument on ultrasound velocity in cancellous bone for the purpose of diagnosing osteoporosis and results on ultrasound velocity and attenuation of leaky surface wave in bone respectively. J. Kushibiki also of Tohoko University discussed results on sound velocity, attenuation and density of a few bovine tissues in the frequency range of 30 to $500 \mathrm{MHz}$ using a buffer rod approach.

There were two sessions consisted of 11 papers with one being an invited paper given by R. Cobbold and T. Tamura from University of Toronto, Canada devoted to blood flow measurements. K. Ferrara, University of California, Davis, presented results to show the advantages of the wideband maximum likelihood estimator over more conventional methods in color Doppler flow mapping. H. Routh of Bio-imaging, now at ATL, described the design and some results on a dual-beam approach for measuring flow transverse to the ultrasonic beams. V. Newhouse of Drexel University and P. Tortoli of University of Florence, Italy, presented theoretical and experimental results to demonstrate that the bandwidth of the Doppler signal is linearly related to flow velocity in transverse Doppler. The invited paper by the University of Toronto group reviewed interesting results obtained in their laboratory on the utilization of a color Doppler flow mapping system for quantitating flow in a mock flow loop. K. Shung of Pennsylvania State University reported recent results on the development of techniques for flow measurements using ultrasonic contrast agents. J. Wilhjelm(Drexel University) discussed experimental results on the FM Doppler systems.

Several papers in a session entitled "Invasive Probes for Imaging and Tissue Characterization" including an invited paper by N. Pandian of Tufts University covered the new exciting frontier in ultrasound: high frequency imaging. G. Lockwood reported experimental results obtained with a scanning acoustic microscope on ultrasound velocity, 
attenuation and backscatter of arterial tissues and blood in the frequency range from 30 to 60 MHz. N. Sanghvi of Indianapolis discussed the development of a high frequency scanning device for imaging cartilage whereas $\mathrm{K}$. Soetanto of Drexel University and T. Matsunaka of Aloka Co., Japan described the developments of two miniature devices for in vivo tissue characterization. N. Pandian, a cardiologist, reviewed the state-of-the-art in intravascular imaging. The advantages of ultrasonic intravascular imaging over conventional imaging methods as well as its clinical applications were addressed in this talk.

\author{
K. Kirk Shung \\ Pennsylvania state University
}

IEEE 1990 Ultrasonics Symposium: Arrays, imaging and scattering.

In Simulation of B-scan images from two-dimensional arrays, F. S. Foster (Ontario Cancer Institute) examined the performance of sparse arrays, randomly sampled throughout the aperture of the transducer. The objective was to design an array with markedly less than full sampling, which could be steered to $\pm 45^{\circ}$. A random sampling of the aperture proved to be the best choice when the beam must be steerable in any direction. The effects of various degrees of sub-sampling on the beam profile and cystic contrast were studied.

C. A. Cain (University of Michigan) presented an intriguing form of ultrasonic imaging, akin to NMR zeugmatography. This replaces the typical mapping of time-of-flight to distance, with pulsed excitation, by a quasi-cw FM imaging mode. The array phasing begins at a large focal length, and low imaging frequency, and the focal length is reduced while the frequency increases as the transmit sequence proceeds. Transmit-receive switching occurs as the focal point reaches the array; the focus then moves deeper as the signal is received. A Fourier transform of the received signal provides reflectivity information in its magnitude, and flow information in its phase.

M. R. Sturgill (Acoustic Imaging) has developed a blood velocity estimator using maximum entropy spectral estimation techniques. Processing backscattered echo signals into velocity estimates for color Doppler is a challenging problem because the signal generally has a broad spectrum, poor signal-to-noise, and a mean frequency close to Nyquist. Current estimation methods, using cross correlations, transforms, or delta phase measurements are prone to systematic errors.

The paper Selcctive rate enhancement of elching mocesses by acoustir. agitation (by Howell, Khuri-Yakub and Kino of Stanford University) showed how etch rates can be speeded up by a factor of four. A sample is exposed to etchant on one side, while a ZnO transducer, fabricated on the other side, supplies up to $1.5 \mathrm{~W}$ of ultrasonic agitation at $400-500 \mathrm{MIIz}$. A variety of pits and trenches were created without the use of masks.

J. Saniie (Illinois Institute of Technology) applied the Wigner distribution, a time-frequency method of signal representation, to ultrasonic flaw detection. The challenge here is the clutter from the material's microstructure which distorts the echoes from a flaw and impairs detection. The performance of this technique was compared to short-time Fourier transforms.

C. M. W. Daft, GE Corporate R\&D Center. 
1990 IEEE Ultrasonics Symposium

Sessions on Medical Signal Processing, Imaging and Inverse Scattering

The session on Medical Ultrasound signal processing focused on the extension of old techniques such as cepstral analysis, impediography, deconvolution and speckle filtering. Results presented by Jorgen Jensen showed modest improvement in B-Scan image quality using 1-dimensional deconvolution processing.

Kirk Beach, a surgeon from the University of Washington, started the session on high resolution/high frame rate imaging by making the controversial statement that, fundamentally, imaging systems have not changed since 1975 . He continued with a sequence of other controversial statements challenging engineers to consider the physician needs in designing future imaging system. Other talks in this session dealt with digital subtraction echography and high resolution imaging. Of particular note were images of the anterior segment of the eye present by F. Stuart Foster of the Ontario Cancer Institute. These images were obtained with a $50-80 \mathrm{MHz}$, low f/number system.

The general theme of the session on advanced imaging systems was the application of novel digital approaches to ultrasound imagers. A VLSI based real-time system capable of high performance, multiple beam forming using digital signal processing methods was presented by the group from General Electric. Also in this session were reports on digital imaging with non-uniform step A/D converters by Hitachi and on volumetric imaging by SRI International. Finally, modest progress was reported in the session on tomography and inverse scattering.

Matthew O'Donnell

University of Michigan 